\title{
CONSIDERACIONES PARA EL USO DE SIMULACIONES EN ENTORNOS VIRTUALES PARA EL APRENDIZAJE DE LAS ESTRATEGIAS DE PROGRAMACIÓN DE COMPUTADORAS
}

\author{
Viviana Berrocal Carvajal ${ }^{1}$
}

\section{Resumen}

El presente documento brinda algunas consideraciones en relación con el uso de las simulaciones para la enseñanza de la programación en entornos virtuales, se consideran factores como el rol del tutor, el rol del estudiante, las condiciones del entorno virtual, las características de las simulaciones y de qué manera podrían apoyar el desarrollo cognitivo al aprender a programar una computadora utilizando diversos lenguajes de programación.

\section{Palabras clave}

-Tutor •Rol del facilitador • Rol del estudiante •Programación de computadores

- Estrategias de mediación y evaluación • Simuladores

\begin{abstract}
This document provides considerations for the use of simulations for teaching programming in virtual environments, we consider the role of tutor, of the student, the terms of the virtual environment, the characteristics of the simulations and how they could support the cognitive development in learning to program a computer using various programming languages.
\end{abstract}

\section{Keywords}

Tutor $\bullet$ Role of the facilitator $\bullet$ Role of the student $\bullet$ Computer Programming

- Strategies of mediation and evaluation $\bullet$ Simulators

\footnotetext{
${ }^{1}$ Máster en Tecnología Educativa. Encargada del Programa de Informática Educativa. Universidad Estatal a Distancia.vberrocal@uned.ac.cr
} 
El sistema educativo debe satisfacer las necesidades de los aprendices, para ello es necesario proveerles medios que los motiven y satisfagan sus expectativas de una educación de calidad y un futuro prometedor, que potencien el uso de sus habilidades cognitivas en el proceso de aprendizaje. En ese sentido, el uso de computadoras y de las Tecnologías de la Información y la Comunicación (TICs) es una de las respuestas ante esta necesidad. La computadora, combinada con otros medios, extiende la capacidad tanto del docente como del estudiante para explorar y descubrir nuevos ambientes de aprendizaje (Barreto \& Gil, 2007).

Desde hace tiempo, se ha incursionado en el uso de TICs en el entorno educativo, sobre todo en los sistemas de educación a distancia. Estas herramientas están siendo agregadas a la gestión del conocimiento y el aprendizaje electrónico orientado hacia el apoyo a los sistemas de aprendizaje formal e informal, las comunidades de aprendizaje y las comunidades que utilizan aplicaciones prácticas dentro de su entorno laboral (Shane, 2007).

Para satisfacer estas demandas, considerando los últimos avances en la tecnología utilizada por Internet y las herramientas para la instrucción, el área de la tecnología educativa se ha convertido en un desafío mayor. Existen tres importantes tendencias en la tecnología educativa: a) la naturaleza cambiante de los alumnos; b) el aumento en la utilización de la tecnología; c) el crecimiento de la tecnología de aprendizaje virtual, sobre todo las simulaciones de actividades han modificado el modelo de enseñanza y aprendizaje (Gupta, 2007).

El sistema educativo se traslada al paradigma basado en la construcción de conocimientos y destrezas de interpretación, análisis y utilización de información. Los docentes propician que el estudiante construya significados mediante experiencias, discusiones, interacciones y reflexiones metacognitivas de forma activa, participativa, inquisitiva e interactiva en un ambiente de 
comunicación, cooperación y colaboración, asociando y transformando lo aprendido con sus esquemas conceptuales tal y como lo indicaron Barreto y Gil (2007) basados en diversos estudios (Aroyo, 2004; Brooks \& Brooks, 1993; Cintron, 2004; Dwyer, 1994; Jeans, 1994; Molina, 2001; Quintero, 1999; Ricen et al., 2001; Yqger, 1991).

En apoyo a los cambios requeridos, las convergencias de las TICs basadas en la gestión de los conocimientos y de los sistemas de aprendizaje virtual ofrecen más control sobre el proceso de aprendizaje del alumno. Los nuevos tipos de interacciones y experiencias de aprendizaje tendrán que ser considerados y desarrollados de acuerdo con las capacidades que ofrece la tecnología. Se requerirá nuevos enfoques y técnicas para lograr el uso de la tecnología a su máximo potencial (Gallagher, citado en Shane, 2007).

El presente documento toma en cuenta las principales consideraciones requeridas para utilizar las simulaciones, de manera que los alumnos aprendan las estrategias requeridas para aprender programación de computadoras utilizando un entorno virtual. Para ello, se consideran los actores involucrados en el proceso de enseñanza y aprendizaje (el entorno virtual, el docente, los estudiantes, la mediación y los recursos utilizados para el aprendizaje), así como las condiciones o requerimientos necesarios para su adecuado empleo dentro de la educación virtual, con el fin de propiciar el logro del objetivo planteado.

A continuación se presentan las principales características de cada uno de los factores necesarios para llevar a cabo la propuesta:

\section{Los Entornos Virtuales}

La integración de las herramientas tecnológicas, así como los métodos instruccionales, son variadas en su alcance y propósito. Por lo tanto, la integración de la tecnología se refiere al 
proceso de determinar qué instrumentos electrónicos y cuáles métodos son adecuados para su aplicación en entornos educativos (Roblyer \& Edwards, citados en Nelson, 2007).

Ares (citado en Gutiérrez, 2006) indicó que:

El e-learning no debe centrarse únicamente en aplicar la tecnología actual a los métodos convencionales de educación. Debe ir más allá y centrarse en determinar cuál es la forma más adecuada para transmitir el conocimiento a aquellos estudiantes que lo precisen... la estrategia para ello consiste en abordar el diseño de un entorno de aprendizaje que constituya una propuesta coherente y adecuada para la formación a distancia a partir de conceptos propios de las teorías pedagógicas. (p. 4)

Sus Características: El aprendizaje que se produce por medio de la transmisión electrónica a través de Internet se ha dado a conocer como aprendizaje en línea o e-learning. En este ambiente, el docente y los estudiantes trabajan a distancia utilizando sus computadores y conexiones a Internet para cumplir con los objetivos curriculares del programa del curso. Por tanto, los estudiantes que no se relacionan en un entorno cara a cara o presencial, se comunican por métodos síncronos o asíncronos. La comunicación asíncrona se refiere a los comentarios, o participaciones, que se envían a un área de un curso en línea o página $W e b$ y no es necesario que las personas estén conectadas a Internet al mismo tiempo, o en tiempo real, con el fin de llevar a cabo un debate o un intercambio de opiniones. La comunicación síncrona permite la interacción de los estudiantes y docentes cuando están conectados al mismo tiempo, o en tiempo real, y los comentarios se consideran tan pronto como los participantes los coloquen en el sitio (Nelson, 2007).

Normas de calidad para entornos virtuales: Para los diversos aspectos involucrados en los entornos virtuales, existen normas que pueden ser definidas como los acuerdos sobre las especificaciones técnicas u otros criterios precisos. Estas son utilizadas como reglas, directrices o definiciones de características, de manera que puedan garantizar que los materiales, productos, procesos y servicios son óptimos para su propósito. En el contexto de aprendizaje en línea, las 
normas son generalmente desarrolladas para su uso en sistemas de diseño y de aplicación para garantizar la interoperabilidad, portabilidad y reutilización. Estos atributos se pretenden aplicar a los sistemas llamados Sistemas de Gestión del Aprendizaje (LMS), al contenido y a los metadatos (Friesen, citado en Shane, 2007).

La calidad viene reflejada en la excelencia de los procesos así como en el logro de los objetivos, de forma tal que, como lo indica Garrison (citado en Gutierrez, 2006):

Los contenidos docentes no determinan por sí solos la calidad de la educación, sino que es el contexto -cómo diseñan los profesores la experiencia educativa, las interacciones inherentes al intercambio, etc.- el que, en último término, distingue unos centros de otros. Una experiencia educativa de calidad consiste en la integración dinámica de contexto y contenidos creada y promovida por un profesor competente tanto en el ámbito pedagógico como en el organizativo. (p. 8)

Entre los factores de calidad en los entornos virtuales de aprendizaje se pueden señalar los siguientes: a) la interacción y el sentimiento de comunidad, la potenciación de la interacción, la acción proactiva, la capacidades interpersonales; b) la elaboración de contenidos multimedia, el Learning Management System (LMS), los factores tecnológicos; c) el modelo de aceptación de la tecnología, la motivación de la elección; d) el sistemas de apoyo, la planificación y el seguimiento del profesor, la retroalimentación rápida, continua y adecuada, los materiales interesantes, la organización de la información, la gestión de la información; e) el número de alumnos; f) la promoción del aprendizaje autónomo, favorecer los estilos de aprendizaje, análisis y crítica de la información; g) la vinculación de la experiencia con el conocimiento previo para favorecer el aprendizaje significativo; h) la utilización variada de métodos de evaluación. 


\section{El rol del docente o facilitador en un entorno virtual}

Con el fin de obtener los beneficios de la integración de la tecnología, las instituciones de educación superior deben mejorar o revisar los métodos utilizados para enseñar la forma en que los docentes integran la tecnología hoy. No es suficiente enseñar a los docentes a utilizar las aplicaciones, deben además entender la manera de planificar su uso en el proceso de aprendizaje. Es necesario trabajar para que los docentes avancen hacia un entendimiento global, los cursos de educación superior se beneficiarían si se alejan de los enfoques didácticos tradicionales y se orientan hacia los centrados en el estudiante y sus enfoques constructivistas. Al utilizar estos enfoques, la tecnología se convierte en un socio en el proceso de aprendizaje y proporciona el vínculo esencial que no sólo motiva a los estudiantes, sino que les ayuda a hacer conexiones entre las tareas que se les pide llevar a cabo y lo que realmente sucede en el mundo real (Nelson, 2007).

Nelson (2007) señala que es importante examinar la manera en que la epistemología constructivista podría apoyar la adquisición de tecnología adecuada en la planificación de capacidades para la integración de la tecnología en las unidades curriculares del profesor y la experiencia en su aplicación. La obtención de la competencia en diversas aplicaciones es importante, pero los docentes deben ser capaces de examinar la forma en que estas aplicaciones se pueden utilizar en el entorno educativo y ser capaces de desarrollar un plan para su utilización eficaz en el curso que imparten.

En el aula tradicional, el docente es visto como el experto que difunde el conocimiento a unos alumnos pasivos. Sin embargo, en un entorno virtual la función cambia a la de un facilitador o guía. El docente debe plantear preguntas de alto nivel, con el propósito de estimular el pensamiento crítico que es necesario para resolver el problema en estudio (Nelson, 2007). 
El profesorado debe estar capacitado para adecuar los objetivos y actividades a las posibilidades formativas del entorno virtual. Para ello, es importante considerar la estructuración de competencias realizada por Marcelo (citado en Gutiérrez, 2006), en las que se especifican las competencias que debe desarrollar el profesorado:

1. Competencia tecnológica: El docente debe contar con un nivel óptimo de autonomía en el manejo de las herramientas informáticas para canalizar la formación, así como en las aplicaciones de Internet, mostrar interés por actualizar sus conocimientos y destrezas, así como la capacidad para simplificar los aspectos procedimentales y tecnológicos.

2. Competencia didáctica: El docente debe tener conocimiento de las teorías del aprendizaje y sus principios, capacidad de adaptación a nuevos formatos, actitud creativa e innovadora para optimizar los recursos, estrategias de comunicación adecuadas, capacidad para diseñar ambientes de aprendizaje pensados para la autodirección y autorregulación, capacidad para crear materiales, plantear tareas que sean relevantes y conocimientos sobre el área de especialidad del curso.

3. Competencia tutorial: El docente debe poseer habilidades de comunicación asertiva, capacidad de adaptación a las condiciones y características de los distintos usuarios, orientación realista de la planificación, mentalidad abierta para aceptar propuestas e introducir reajustes, capacidad de trabajo y constancia.

Según lo indicado por Gutiérrez (2006):

El cometido del profesorado consiste en preparar a los alumnos para que no dejen de serlo nunca, ayudando a manejar la gran cantidad de información de que disponen, desarrollar el pensamiento crítico y las capacidades de autoaprendizaje que les serán útiles a lo largo de su vida. De esta manera, el profesor es el arquitecto y, a la vez, un animador para diseñar, dirigir y alimentar las situaciones transaccionales educativas, las 
cuales deben darse en una relación equilibrada y funcional a la vez que coherente con los resultados previstos y en relación con las capacidades y necesidades de cada estudiante. (p.5)

\section{El rol del estudiante en un entorno virtual}

El estudiante es quien debe asumir su propio proceso de aprendizaje de manera tal que se promueva su participación activa, dentro de lo que se destaca el desarrollo de opiniones que debe compartir con sus compañeros y profesores, la posibilidad de realizar trabajos en equipo aunque se encuentren a millas de distancia y con horarios de trabajo muy diferentes.

Los estudiantes que participan en el entorno virtual deben presentar según Simonson, Smaldino, Albright \& Zvacek (2006) las siguientes características: a) se sienten cómodos con el aprendizaje y la interacción con otros a través de la tecnología; b) están dispuestos a interactuar eficazmente con sus compañeros y participar en los procesos grupales; c) tienen la posibilidad de comunicarse de manera efectiva a través de la escritura; d) tienen la habilidad de replantear sus ideas antes de responder; se automotivan, son autodisciplinados y buenos administradores de su tiempo; e) tienen la posibilidad de realizar el auto-estudio, según sea necesario, y construyen los conocimientos para llenar las lagunas en la información que pueden ocurrir en un sistema de aprendizaje virtual; f) tienen la posibilidad de administrar el tiempo que requiere el curso; g) su mente está abierta para compartir sus experiencias de vida, de trabajo y educativas, como parte del proceso de aprendizaje; h) están dispuestos a tomar riesgos y ser creativos; i) están dispuestos a "hablar" si surgen problemas; j) tienen la posibilidad de hacer caso omiso de las distracciones y centrarse en el propio entorno de aprendizaje; k) tienen un compromiso con la creencia de que la más alta calidad, puede llevarse a cabo en ausencia de un aula tradicional; 1) son lo suficientemente flexibles para aceptar la enseñanza y el aprendizaje en un contexto diferente y tener la capacidad de adaptarse con éxito al aprendizaje en un entorno en línea.

\section{Otras condiciones necesarias para el aprendizaje virtual}

Además de los aspectos considerados anteriormente, existen otras condiciones que se deben tomar en cuenta para garantizar el éxito del proceso. 
1. Políticas institucionales: se debe contar con el establecimiento de políticas institucionales para la instrucción en línea que permitan clarificar las condiciones y requerimientos del proceso de enseñanza y aprendizaje en un entorno virtual.

2. Soporte Institucional: es importante contar con soporte o apoyo en aspectos técnicos en el uso de recursos y con asesoramiento en cuanto a las estrategias utilizadas en los entornos de aprendizaje virtual. Este asesoramiento debe estar presente en las etapas de diseño y durante la ejecución de los cursos (Tallent-Runnels et al., 2006).

\section{Las simulaciones}

La simulación es una avanzada tecnología de información, utilizada para representar situaciones de la realidad, con su ayuda los participantes aprender mediante la observación de las situaciones predefinidas. En algunos casos, este tipo de aplicaciones también posibilita que los participantes interactúen con la aplicación en un entorno controlado, sensorial y proporcionar comentarios acerca de las acciones, permite también el aprendizaje reflexivo sobre las consecuencias de la propia acción (Grupta, 2007).

Simulaciones en el entorno educativo: Las simulaciones en el entorno educativo son definidas como programas educativos que incorporan modelos acompañados de objetivos de aprendizaje para la comprensión de la situación planteada. Existen varias definiciones que las describen como creaciones sintéticas o falsificadas, una aproximación artificial de la realidad, algo que crea la realidad de un lugar de trabajo, o modelos matemáticos que permiten la predicción y visualización en el tiempo, además, son consideradas como las únicas estrategias instruccionales que son coherentes y repetibles en un contexto educativo, son un proceso cíclico de aprendizaje, uno de los más eficaces modelos de la enseñanza tal y como lo menciona Shane (2007) basado 
en diversos autores (Alessi, 2000; Prensky, 2001; Norton \& Sprague, 2001; Saunders, 1997; Saleh, 2005).

Las simulaciones en la educación se consideran importantes instrumentos para apoyar el aprendizaje, tanto en áreas científicas como de letras. Sin embargo, existe confusión sobre el alcance y la definición por lo general debido a la terminología. El mismo tipo de simulación a menudo es descrito mediante muchos términos. Por ejemplo, micromundos, simuladores de vuelo, de negocios, de gestión; el aprendizaje y el medio ambiente son términos que a veces describen el mismo tipo de simulación. Asimismo, dos simulaciones con el mismo nombre pueden tener una funcionalidad y un tipo distintos (Maier \& Grobler, citados en Shane, 2007).

La diversidad se pone de manifiesto por los efectos que rodean el desarrollo y el despliegue de las simulaciones en el contexto de aprendizaje. Entre los objetivos de las simulaciones se pueden citar el hecho de que el aprendizaje y la forma realizan la función con un equipo (médico o de vuelo), la comprensión de los sistemas a través de la exploración (laboratorios virtuales o modelos) y prácticamente cualquier aplicación donde la disciplina y el orden superior de aprendizaje son importantes. Las simulaciones pueden facilitar la formación de un alumno en un entorno virtual en el cual el planteamiento o realización de ejercicios prácticos es demasiado costoso o peligroso en la realidad, como los entornos tóxicos o de alta fidelidad, como los simuladores de vuelo (Shane, 2007).

Cuando las simulaciones son orientadas hacia el aprendizaje, si están bien diseñadas pueden tener un alto nivel de transferencia del aprendizaje ideal en la educación y la formación. La transferencia es considerada como la capacidad de un alumno para aplicar lo que se ha aprendido en una situación de aprendizaje de manera rápida y eficaz a otras situaciones de la vida real (Driscoll, citado en Shane, 2007). 
En el entorno educativo se utilizan básicamente dos tipos de simulaciones:

1. Modelo de simulación: Es en el que se ofrece una orientación para representar diferentes aspectos relevantes a un modelo de simulación para la instrucción, en el que se permite la representación de los conceptos que se pueden utilizar en un contexto de instrucción para el razonamiento sobre el comportamiento del sistema.

2. Modelo de instrucción: Un entorno de desarrollo de simulación de instrucción debe apoyar el uso de la terminología similar, las fórmulas y las metáforas visuales para adaptarse a la elección de los libros de texto existentes. Este tipo de simulaciones no pretenden sustituir las clases formales, sino más bien se utilizan para mejorar ciertas partes de la misma dinámica con las representaciones de procesos difíciles de reproducir en el ambiente educativo. En el ámbito de estos objetivos de instrucción, los docentes determinan el material de aprendizaje y cómo se utiliza para probar el cumplimiento de los objetivos específicos de instrucción. Estos objetivos pueden ser empleados en el contexto de las simulaciones instruccionales para reducir la brecha entre la teoría y la práctica. Estas prácticas pueden ayudar a formar los procedimientos y aprender sobre la vida real, pero a menudo no logran conectarse a conocimientos teóricos (Kuyper, How \& Jong, 2001).

Este tipo de aplicaciones debe además contemplar algunas consideraciones éticas y psicológicas, entre las que se destacan el potenciar un liderazgo, el manejo del error como fuente de aprendizaje y los principios de valores éticos y morales en los estudiantes que hacen uso de ellas en su entorno (Smith, 2008).

Las simulaciones instruccionales se pueden considerar un modelo pedagógico, ya que del contenido de los programas y la elección de la metodología (o metodologías) logran los resultados del aprendizaje mediante el despliegue de los procedimientos específicos o estrategias 
pedagógicas. Son consideradas como un modelo para ayudar a los estudiantes a comprender y obtener sus modelos del mundo en el contexto de la instrucción (Grimmitt y Driscoll, citados en Shane, 2007).

\section{Aspectos por tomar en cuenta para el diseño de simulaciones en entornos educativos}

Cuando se plantea el diseño de una simulación para un entorno educativo o comercial, se toma como un proceso de diseño que consta de tres pasos principales: la especificación de los requisitos a partir de los objetivos del diseño; la construcción de bloques basados en la aplicación sobre la base de las necesidades y la evaluación de la aplicación en función de los objetivos. Este es un proceso iterativo, llamado modelo evolutivo (Kuyper et al., 2001).

La introducción de simulaciones de instrucción en el plan de estudios es parte de una tendencia basada hacia la incorporación de las TICs en el aprendizaje y encaja con opiniones sobre la educación que defienden el aprendizaje independiente (Kuyper et al., 2001).

Con este propósito, se están incorporando varios tipos de simulaciones, según lo indica Sánchez, Dormido y Morilla (s.f.), entre los que se destacan:

1. Simulación remota: es un laboratorio virtual que se está desarrollando para la realización de simulaciones dinámicas e interactivas a través de Internet (Web-based simulation).

2. Telepresencia: se está desarrollando un entorno de telepresencia para el empleo remoto de ciertas plantas de entrenamiento, lo cual posibilita, en la medida que la tecnología lo permite, transmitir a los estudiantes las sensaciones y responsabilidades que tendrán cuando se desempeñen en el entorno laboral.

3. Visualización remota: este sistema demanda la existencia de una comunicación visual y auditiva con la planta sobre la que se estén realizando las experiencias. El objetivo es la creación de un entorno de trabajo que permita la supervisión visual remota del proceso que se está 
realizando. Permite realizar labores de teleasistencia médica, teleoperaciones quirúrgicas, o de controles en procesos de ingeniería (Sánchez et al., s.f.).

Sistemas híbridos en ciencia y tecnología educativa: En este sistema, los estudiantes crean modelos en el computador y ejecutan simulaciones para solucionar problemas dados, construyen así su conocimiento y mejoran sus modelos mentales sobre el sistema natural y tecnológico (Forrester y Jackson \& Yarger citados en Levin y Levin, 2002). Este sistema proporciona una metodología sistemática para el diseño del mismo, cubre una pluralidad que incluye componentes naturales y tecnológicos, las tareas del control se formulan y se estudian naturalmente con los modelos híbridos y proporciona un contexto significativo para enseñar conceptos fundamentales de las matemáticas discretas, de la informática y del diseño digital. Estos conceptos ahora se enseñan principalmente en la ingeniería y las clases de la informática y por lo tanto, se limitan a un grupo pequeño de profesionales. Sin embargo, la influencia de estos conceptos en el mundo moderno debe hacerles parte de la educación general, en el marco de la ciencia y de la tecnología educativa (Kim et al., 2000).

Para apoyar estos procesos se utilizan acciones como:

1. Monitorización on-line del proceso: En donde hay una visualización directa y en tiempo real de cómo las acciones de control son efectuadas. De esta forma, la realimentación que el estudiante obtiene no sólo se basa en los resultados, sino también en la observación directa del proceso.

2. Comprobación en línea del proceso: por medio de una grabación en video de toda o parte de la experiencia en la planta, de manera que se pueda volver a estudiar la experiencia.

3. Refuerzo de conceptos: por medio de un video de alguna experiencia previa se puede reforzar algún concepto teórico. 
4. Planteamiento de ejercicios: presentación visual de alguna situación, para que el estudiante la revise con anterioridad y piense en una manera de solucionar la situación, en caso de que se le presente durante su sesión de trabajo (Sánchez et al., s.f).

Seguimiento y evaluación: El fin que se persigue con las simulaciones es mejorar los conocimientos del alumno sobre la materia en cuestión, lo cual no puede lograrse sin que el estudiante reciba una realimentación sobre la validez del trabajo desarrollado dentro del laboratorio, así como que el docente conozca el grado de avance experimentado por el alumno. Por las razones citadas, durante todo el laboratorio virtual debe existir un módulo de seguimiento y evaluación del alumno que le permita autodirigirse en su proceso de aprendizaje, indicándole los puntos sobre los que necesita un mayor refuerzo en su estudio.

Al mismo tiempo, el módulo debe servir al profesor tanto para comprobar que el proceso de aprendizaje que lleva asociado el estudiante es el correcto (seguimiento puntual), como para conocer si se han logrado los objetivos perseguidos tras el estudio completo de la asignatura (seguimiento global). De acuerdo con lo anterior, algunas de las tareas que debe llevar a cabo el módulo de seguimiento y evaluación son: (a) generación y corrección de test sobre la materia; (b) programación de experimentos para el refuerzo y fijación de determinados conceptos; (c) evaluación del resultado de los experimentos y (d) seguimiento continuo de las simulaciones y del estado del laboratorio (Sánchez et al., s.f).

\section{Orientaciones para el uso de simulaciones en un contexto educativo}

Si se toma en cuenta que el diseño instruccional considera aportaciones de diferentes campos tales como las teorías generales del aprendizaje y del diseño instruccional; las investigaciones en la didáctica de las ciencias; los entornos educativos multimedia y espacios colaborativos de 
aprendizaje, etcétera, se puede deducir una serie de directrices que van a orientar el diseño de entornos de aprendizaje basados en simulaciones informáticas:

1. Las simulaciones deben ser usadas para promover un aprendizaje basado en la investigación de los alumnos.

2. En estos procesos de enseñanza y aprendizaje los alumnos tienen un papel activo, al considerar sus estilos y ritmos de aprendizaje, así como las dificultades de los alumnos.

3. Las actividad investigadora de los alumnos se potencia en un ambiente colaborativo.

4. El proceso investigador de los alumnos ha de estar orientado mediante la adecuada realimentación.

5. El diseño de las actividades basadas en simulaciones debe tener en cuenta su carácter multimedia. Al respecto, Mayer y Moreno (2002; citados en García \& Gil, 2006), han desarrollado una teoría cognitiva del aprendizaje multimedia apoyada en las teorías de codificación dual, carga cognitiva y aprendizaje constructivista. Los mensajes multimedia deben cumplir con cuatro principios: a) contigüidad: se aprende mejor cuando la animación y la narración se presentan al mismo tiempo; b) coherencia: mejor aprendizaje cuando no hay que procesar imágenes o palabras extrañas en la memoria de trabajo; c) modalidad: es más conveniente presentar la palabra en forma de narración que como texto escrito y d) redundancia: el aprendizaje se dificulta cuando se presentan al mismo tiempo narración y texto escrito.

6. El uso de las simulaciones debe ser coherente con un planteamiento constructivista del proceso de enseñanza y aprendizaje, que tome en cuenta los conocimientos previos de los alumnos, el contexto social y el problema que ha de ser resuelto. El proceso instructivo, por tanto, se concreta en proveer a los alumnos de una situación colaborativa en la que tengan los medios y las oportunidades de construir nuevos aprendizajes. Para ello se debe: a) partir de 
situaciones problemáticas capaces de provocar interés; b) analizar cualitativamente la situación y proponer un plan para abordarla; c) usar estrategias coherentes con el trabajo científico para resolver el problema, tales como el plantear hipótesis, elaborar estrategias, analizar resultados y cotejarlos con las previsiones; utilizar reiteradamente los nuevos conocimientos en diversas situaciones con el objeto de consolidarlos y crear cuerpos coherentes con ellos.

7. El tratamiento de los problemas ha de ser global ya que debe abarcar situaciones sobre la concepción del mismo y los aspectos involucrados en su solución.

8. Se debe provocar un entorno de aprendizaje constructivista (EAC) para diseñar la instrucción educativa, articulado en torno al tratamiento de cuestiones, proyectos, problemas o ejemplos de interés para los alumnos que generan un proceso investigador en el que las aplicaciones informáticas son utilizadas de formas diversas tales como el acceso a información, herramientas cognitivas y de comunicación, etcétera (García et al., 2006).

En la Figura 1, planteada por García et al. (2006), se muestran las principales relaciones que se derivan de un proceso de enseñanza y aprendizaje basado en simulaciones informáticas, se evidencia que su utilización potencia el aprendizaje significativo por medio de la participación activa de los estudiantes, en un entorno constructivista que desarrolla procesos de investigación para la resolución de las situaciones de aprendizaje planteadas. 


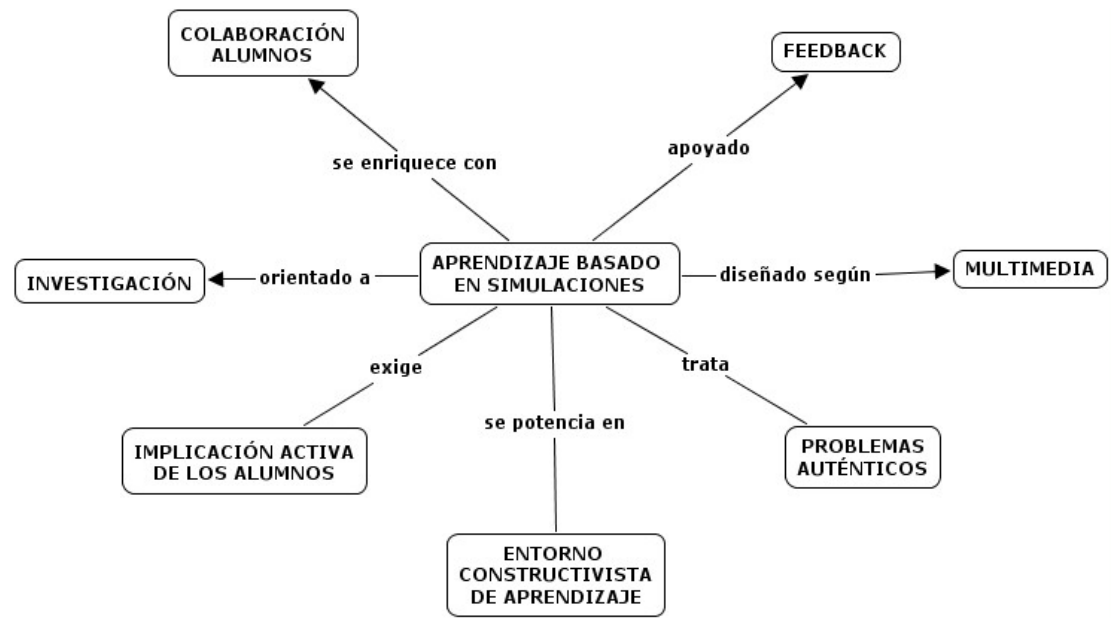

Figura 1. Esta figura representa las relaciones que se establecen por medio del aprendizaje basado en simlaciones.

\section{El uso de las simulaciones para la enseñanza de la programación de computadoras}

Los estudiantes de programación a menudo presentan gran dificultad para entender lo que está haciendo el computador realmente cuando ejecuta un programa, es esta carencia de la comprensión uno de los principales problemas que debe ser superado antes de que el futuro programador se sienta cómodo escribiendo programas para computadoras. Desde 1983, este planteamiento ha sido objeto de investigaciones, dentro de las que se destacan Domingue y Mulholland, 1998; Eisenstadt y Breuker, 1992, Soloway, Bonar y Ehrlich, 1983; y Rajan, 1992 (citados en Rowe, 2000), coincidieron en que una posible solución del problema era forzar las experiencias diarias de los estudiantes a la ejecución de tareas repetidoras en el marco de un lenguaje de programación, ya que estos lenguajes hacen cosas de una manera no-intuitiva; también indicaron que había una carencia de la comprensión de lo que realizan los lenguajes de programación cuando se ejecuta un programa. Sugieren que una "máquina teórica", vista como una representación, en cierta forma, del computador en sí mismo, ayudaría a entender su funcionamiento y sería de gran ayuda a los principiantes; esta máquina teórica debe "tener una vista dinámica del algoritmo en la acción". 
Se han desarrollado propuestas para la visualización del software centrado en la enseñanza de los algoritmos, también se ha trabajado en el desarrollo de software para la enseñanza de la programación. Estas propuestas, sin embargo, no se ocupan del problema de visualizar las operaciones de un programa sobre una base paso a paso tal y como lo indicaron Lewis y Mulley, en Rowe y Thorburn (2000), ya que han producido un compilador “principiante-cómodo" que incluyen alertas y mensajes de error de una manera más informativa. También estos autores recogieron estadísticas sobre los tipos de errores más comunes de los programadores principiantes y observaron que muchos de estos errores eran debido a una carencia de la comprensión de la manera en que el programa maneja los datos.

Lorenzo, Badre y Stasko (citados en Rowe y Thorburn, 2000), proporcionaron animaciones sobre algoritmos a los estudiantes y al evaluarlos encontraron que estas prácticas permitieron que ellos crearan sus propios ejemplos, los cuales eran más valiosos que los creados por los estudiantes que trabajaron con ejemplos fijos. También hallaron que las animaciones eran especialmente útiles para lograr en los estudiantes una comprensión más profunda de los conceptos usados en un algoritmo y facilitaba el hecho de que ellos pudieran explicar cómo un algoritmo trabajó, y poder seguir los pasos del algoritmo mecánicamente para producir un resultado. Por ello, se considera que el uso de las simulaciones y las TICs podrían ayudar a solucionar estos problemas y potenciar el uso de los recursos computacionales en los procesos de aprendizaje, de manera que los estudiantes subsanen sus dificultades y entiendan los conceptos involucrados en la elaboración de los algoritmos de programación en los que se basa la programación de los computadores. 


\section{CONCLUSIONES}

Debido a la gran cantidad de información que se encuentra disponible, a la rapidez con que ésta se produce, es inminente contar con herramientas para establecer mecanismos de interacción entre el contenido, los estudiantes y los docentes, con el fin de facilitar este proceso se recurre al uso de las diversas herramientas tecnológicas que permiten provocar procesos de aprendizaje activos centrados en el estudiante (Scott, 2007).

Cuando se recurre al uso de las simulaciones y las TICs en un proceso de aprendizaje, a parte de las potencialidades de las herramientas, se deben considerar otros factores, dentro de los que se destacan el rol del tutor y el del estudiante, las estrategias de mediación y evaluación, la calidad de los productos y los procesos para que su incursión en el proceso educativo realmente favorezca el proceso de enseñanza-aprendizaje y se permita que los estudiantes involucrados logren un aprendizaje significativo. El aprendizaje y la actividad son considerados inseparables y están incorporados en el uso de herramientas; los objetos de aprendizaje y los recursos deberían apoyar las complejas interacciones necesarias para el aprendizaje significativo. Tanto el proceso de aprendizaje como las actividades de evaluación se basan en métodos pedagógicos o técnicas de formación que permiten el desarrollo de un proceso de aprendizaje significativo, para lo cual las simulaciones brindan un marco que permite el logro de los objetivos planteados en el proceso de enseñanza y aprendizaje. Las simulaciones también proporcionan un auténtico y eficaz ambiente de evaluación, ya que se pueden demostrar y aplicar lo que se ha aprendido.

En el aspecto formativo, las simulaciones se pueden utilizar para ayudar a los estudiantes a reflexionar y dar forma a sus conocimientos y habilidades; así como espacios de exposición y ejecuciones del entendimiento. Esta herramienta apoyada por las TICs permite un proceso de 
aprendizaje que garantiza la interacción y construcción del aprendizaje significativo por parte de los estudiantes.

Por lo tanto, las estrategias que proporciona la tecnología instruccional, a través de la configuración del mundo real, pueden resultar eficaces. Por consiguiente, el aprendizaje basado en problemas $(\mathrm{PBL})$ en el entorno en línea, puede proveer el escenario apropiado para que los docentes utilicen la tecnología como un socio en el proceso educativo, ya que con este método se trata de estimular el aprendizaje de la información, enseña a la vez un método de enfocar y una actitud hacia la resolución de problemas.

Por sus características y potencialidades, las simulaciones utilizadas en un entorno virtual son excelentes instrumentos de evaluación para certificar si alguien puede resolver problemas o realizar análisis de las actividades, por ello su uso puede solucionar los problemas encontrados en los estudiantes principiantes de programación. 


\section{REFERENCIAS}

Barreto, L. (2007). Uso de las tecnologías de información y comunicación: las visiones y experiencias tres maestros de educación superior. Disertación Doctoral, Universidad de Puerto Rico.

García, A.; Gil M. (2006). Entornos constructivistas de aprendizaje basados en simulaciones informáticas. Revista Electrónica de Enseñanza de las Ciencias, 5 (2).

Gupta, S. (2007). Simulations and technology training: A Need to Revisit Training Goals. Business Education Forum, 62 (2), 51-4.

Gutiérrez, E. (2006). Nuevos papeles del profesorado de educación superior en entornos virtuales. V Congreso Internacional "Educación Y Sociedad". Universidad de Granada. Recuperado el 15 de marzo de 2008, de http://congreso.codoli.org/area_2/GutierrezSantiuste.pdf.

Kim, T.; Jackson, D.; Yarger, D. (2000). Principles for the design and use of simulations in science learning as exemplified by a prototype microworld. The Journal of Computers in Mathematics and Science Teaching, 19 (3), 237-52.

Kuyper, M.; Hoog, R.; Jong, T. (2001). Modeling and supporting the authoring process of multimedia simulation based educational software: a knowledge engineering approach. Instructional Science, 29 (4/5), 337-59.

Levin, T.; Levin, I. (2002). Sistemas híbridos que modelan en ciencia y tecnología que aprenden. El diario de ordenadores en las matemáticas y la ciencia que enseñan, 21 (4), 313-330.

Nelson, Erik T. (2007). Effects of online problem-based learning on teachers' technology perceptions and planning. Disertación Doctoral, Capella University.

Rowe, G.; Thorburn, G. (2000). VINCE--an on-line tutorial tool for teaching introductory programming. British Journal of Educational Technology British Journal of Educational Technology, 31 (4), 359-69.

Sánchez, J.; Dormido, S.; Morilla, F. (s.f). Laboratorios virtuales y remotos para la práctica a distancia de la automática. Centro Virtual Cervantes. Recuperado el 5 de marzo de 2008, en: http://cvc.cervantes.es/obref/formacion_virtual/campus_virtual/sanchez.htm.

Scott, W. (2007). A case study of the adoption of a technology based innovation in an urban school district: An e-portfolio initiative. Disertación Doctoral.UMI. Norman Oklahoma.

Shane Gallagher, P. (2007). Assessing SCORM 2004 for its affordances in facilitating a simulation as a pedagogical model. Disertación Doctoral, George Mason University. 
Simonson, M.; Smaldino, S.; Albright, M. y Zvacek, S. (2006). Teaching and learning at a distance: Foundations of distance education ( $3^{\text {ra }}$ ed.). Upper Saddle River, NJ: Prentice Hall.

Smith, D. (2008). Simulations as action learning devices. Learning and Leading with technology, p. 30-31.

Tallent-Runnels, M.; Thomas, J.; Lan, W.; Cooper, S.; Ahern, T.; Shaw, S. et al. (2006).

Teaching courses online: A review of the research. Review of Educational Research, 76 (1), 93-135. 